\title{
Non-negative Wigner functions in prime dimensions
}

\author{
D. Gross \\ Institute for Mathematical Sciences, Imperial College London, London SW7 2BW, UK and \\ QOLS, Blackett Laboratory, Imperial College London, London SW7 2BW, UK *
}

(Dated: July 30, 2018)

\begin{abstract}
According to a classical result due to Hudson, the Wigner function of a pure, continuous variable quantum state is non-negative if and only if the state is Gaussian. We have proven an analogous statement for finite-dimensional quantum systems. In this context, the role of Gaussian states is taken on by stabilizer states. The general results have been published in [D. Gross, J. Math. Phys. 47, 122107 (2006)]. For the case of systems of odd prime dimension, a greatly simplified proof can be employed which still exhibits the main ideas. The present paper gives a self-contained account of these methods.
\end{abstract}

\section{INTRODUCTION}

The Wigner distribution establishes a correspondence between quantum mechanical states and real pseudoprobability distributions on phase space. 'Pseudo' refers to the fact that, while the Wigner function resembles many of the properties of probability distributions, it can take on negative values. It is therefore of interest to characterize those quantum states that are classical in the sense of giving rise to non-negative phase space distributions.

For the case of pure states described by vectors in $\mathcal{H}=L^{2}(\mathbb{R})$, the resolution of this problem was given by Hudson in Ref. [1], and later extended to multipleparticles by Soto and Claverie (Ref. [2]).

Theorem 1. (Hudson, Soto, Claverie) Let $\psi \in L^{2}\left(\mathbb{R}^{n}\right)$ be a state vector. The Wigner function of $\psi$ is nonnegative if and only if $\psi$ is a Gaussian state.

By definition, a vector is Gaussian if and only if it is of the form

$$
\psi(q)=e^{2 \pi i(q \theta q+x q)},
$$

where $x \in \mathbb{R}^{n}$ and $\theta$ is a symmetric matrix with entries in $\mathbb{C}[20]$.

It is our objective to prove that the situation for discrete quantum systems is very similar, at least when the dimension of the Hilbert space is odd. The following Theorem states the main result.

Theorem 2. (Discrete Hudson's Theorem) Let $d$ be odd and $\psi \in L^{2}\left(\mathbb{Z}_{d}^{n}\right)$ be a state vector. The Wigner function of $\psi$ is non-negative if and only if $\psi$ is a stabilizer state.

Given that $\psi(q) \neq 0$ for all $q$, a vector $\psi$ is a stabilizer state if and only if it is of the form

$$
\psi(q)=e^{\frac{2 \pi}{d} i(q \theta q+x q)},
$$

where $x \in \mathbb{Z}_{d}^{n}$ and $\theta$ is a symmetric matrix with entries in $\mathbb{Z}_{d}$.

*Electronic address: david.gross@imperial.ac.uk
In the previous theorem, $\mathbb{Z}_{d}:=\{0, \ldots, d-1\}$ denotes the set of integers modulo $d$.

It turns out that, although the formulation of Hudson's result carries naturally over to finite dimensional systems, the respective proofs are radically different. The original argument relies crucially on function theory, which is of course not available in the setting that this paper addresses.

Recently, Galvao et. al. took a first step into the direction of classifying the quantum states with positive Wigner function (see Ref. 3] ). To explain the relationship of their results to the present paper, we have to comment shortly on two different approaches to defining discrete Wigner functions. On the one hand, it has long been realized that Wigner's definition carries over naturally to discrete odd-dimensional systems (Ref. 4, 5, 6, 7, 8, 9, 10, 11, 12, 13, 14 and Section III). This approach is the one used in the present paper. On the other hand, Gibbons, Hoffmann, and Wootters listed a set of axioms that candidate definitions have to fulfill in order to resemble the properties of the well-known continuous case (Ref. [13]). Let us call functions that fall into this class generalized Wigner functions. The characterization does not specify a unique solution: for a $d$ dimensional Hilbert space, there exist $d^{d-1}$ distinct generalized Wigner functions. The construction of Gibbons et. al. has been described only for the case where $d$ is the power of a prime.

If the dimension of the Hilbert space is of the form $d^{n}$, a second ambiguity arises. We are free to conceive such a space either as being associated to a system of $n$ constituents, each of dimension $d$, or to a single one of dimension $d^{n}$. While the Wigner function is the same for both cases, the set of stabilizer states is not (see Refs. [5, 12]). Indeed, the 'single-particle' stabilizer states turn out to be a proper subset of the 'multiple-particle' ones. As a striking example, the generalized Bell and GHZ states nor are not stabilizer states on single $d^{2}$ or $d^{3}$-dimensional systems.

In Ref. [3] it was proved that a state of a single-particle system of prime-power dimension is a stabilizer state if and only if all its generalized Wigner functions are nonnegative. The authors aim to establish necessary requirements for quantum computational speedup. Indeed, if 
the Wigner function of a quantum computer is positive at all times, then it operates only with stabilizer states and hence offers no advantage over classical computers, by the Gottesman-Knill Theorem (Ref. 15]).

For the case of pure states, our results imply the ones of Ref. [3] and exceed them in two ways. Firstly, we show that it suffices to check positivity for a single definition of the Wigner function, as opposed to $d^{d-1}$ ones. Secondly, our statements hold for multiple-particle systems, which constitute the proper setting for both quantum computation and the Gottesman-Knill Theorem. On the other hand, Ref. [3] makes assertions about mixed states and qubit systems, which are not covered by our findings.

Our general results have been published in Ref. [5]. However, the proof is rather involved. Many technicalities arise due to the fact that for non-prime $d$, arithmetic modulo $d$ lacks the desirable properties of finite fields. Our aim in writing Ref. [5] was to achieve the broadest possible generality in spite of these difficulties. The downside of this approach is that core ideas of the argument are obscured by technical issues. The present paper employs a different method of proof, which is available only for systems of odd prime dimension. For this special case, the main result of Ref. [5] can be obtained using only a fraction of the space. It is our hope that this paper makes the ideas accessible to a wider audience.

The next section summarizes further findings contained in Ref. [5]. We go on to recall the definition and properties of discrete Wigner functions in Section [II] Section IV is devoted to a complete proof of the easiest special case of Theorem 2. that being given by a single particle on a Hilbert space of prime dimension.

\section{FURTHER RESULTS AND IMPLICATIONS}

It is natural to ask how Hudson's results generalize to mixed states. Certainly, mixtures of Gaussian states are positive on phase space and Narcowich in Ref. [16] conjectured that all such quantum states are convex combinations of Gaussian ones. Bröcker and Werner refuted the conjecture by giving a counter-example (Ref. [17]). We show in Ref. [5] that the situation is similar in the finite setting.

Further, we show how to lift the ambiguity in the axiomatic characterization of Wigner functions by requiring Clifford covariance, note that a unitary operator preserves positivity if and only it is a Clifford operation, discuss the relation of various ways to introduce Wigner functions and stabilizer states in dimensions of the form $d=p^{n}$, and give an explicit account on the connection between stabilizer states and Gaussian states.

\section{WIGNER FUNCTIONS}

This section provides a very superficial introduction to discrete Wigner functions. We allow ourselves to refer the reader to Refs. [5] for further details. In what follows $d$ denotes an odd prime. All integer arithmetic in this paper is implicitly assumed to be $\bmod d$. The symbol $2^{-1}=(d+1) / 2$ is the multiplicative inverse modulo $d$. All state vectors are elements of the Hilbert space $\mathcal{H}$ spanned by $\{|0\rangle, \ldots,|d-1\rangle\}$. Lastly, $\omega=e^{\frac{2 \pi}{d} i}$ is a $d$ th root of unity.

The relations

$$
x(q)|k\rangle=|k+q\rangle, \quad z(p)|k\rangle=\omega^{p k}|k\rangle
$$

define the shift and boost operators respectively. The most central element in the theory are the Weyl operators (in quantum information also known as the generalized Pauli operators) given by

$$
w(p, q)=\omega^{-2^{-1} p q} z(p) x(q) .
$$

The characteristic function of an operator $\rho$ is given by the expansion coefficients of $\rho$ in terms of the Weyl operators

$$
\Xi_{\rho}(\xi, x)=\frac{1}{d} \operatorname{tr}\left(w(\xi, x)^{\dagger} \rho\right) .
$$

We define the Wigner function to be the symplectic Fourier transform of the characteristic function:

$$
W_{\rho}(p, q)=\frac{1}{d} \sum_{\xi, x \in \mathbb{Z}_{d}} \omega^{p \xi-q x} \Xi_{\rho}(\xi, x) .
$$

It is a tedious yet straight-forward computation to show that the Wigner function of a pure state is given by

$$
\begin{aligned}
W_{\psi}(p, q) & :=W_{|\psi\rangle\langle\psi|}(p, q) \\
& =\frac{1}{d} \sum_{\xi \in \mathbb{Z}_{d}} \omega^{-\xi p} \psi\left(q+2^{-1} \xi\right) \bar{\psi}\left(q-2^{-1} \xi\right) .
\end{aligned}
$$

If $S$ is a $2 \times 2$-matrix with elements in $\mathbb{Z}_{d}$ and determinant 1 , then there exists a unitary operation $\mu(S)$ (the Weil [18] 21] or metaplectic representation of $S$ ) such that

$$
\mu(S) w(p, q) \mu(S)^{\dagger}=w(S(p, q)) .
$$

The Wigner function is covariant in the sense that, if $\rho^{\prime}=\mu(S) \rho \mu(S)^{\dagger}$, then

$$
W_{\rho^{\prime}}(p, q)=W_{\rho}(S(p, q)) .
$$

Similarly, the Weyl operators induce translations of the Wigner function. Letting $\rho^{\prime}=w\left(p^{\prime}, q^{\prime}\right) \rho w\left(p^{\prime}, q^{\prime}\right)^{\dagger}$, it holds that

$$
W_{\rho^{\prime}}(p, q)=W_{\rho}\left(p+p^{\prime}, q+q^{\prime}\right) .
$$

The Clifford group is the set of unitary matrices that send Weyl operators to Weyl operators under conjugation [22]. Every Clifford mapping is of the form $w(p, q) \mu(S)$ and hence preserves positivity of the Wigner function.

Finally, stabilizer states are the images of the computational basis states under the action of the Clifford group. 


\section{MAIN THEOREM - SINGLE PARTICLES IN PRIME DIMENSIONS}

Define the self correlation function

$$
K_{\psi}(q, x)=\psi\left(q+2^{-1} x\right) \bar{\psi}\left(q-2^{-1} x\right)
$$

and note that the Wigner function obeys

$$
W(p, q)=\frac{1}{d} \sum_{x} \omega^{-p x} K_{\psi}(q, x) .
$$

Recall that the Fourier transform $\hat{f}$ of a function $f$ : $\mathbb{Z}_{d} \rightarrow \mathbb{C}$ is defined to be $\hat{f}(x)=1 / d \sum_{q} \omega^{-q x} f(q)$. Therefore, for a fixed $q_{0}, W\left(p, q_{0}\right)$ is the Fourier transform of $K\left(q_{0}, x\right)$. Hence $W$ is non-negative if and only if the $d$ functions $K\left(q_{0}, \cdot\right)$ have non-negative Fourier transforms.

In harmonic analysis, the set of functions with nonnegative Fourier transforms is characterized by a wellknown theorem due to Bochner. We state an elementary version of Bochner's Theorem, along with a variation for subsequent use.

Theorem 3. (Variations of Bochner's Theorem) Consider a function $f: \mathbb{Z}_{d} \rightarrow \mathbb{C}$. It holds that

1. The Fourier transform of $f$ is non-negative if and only if the matrix

$$
A^{x}{ }_{q}=f(x-q)
$$

is positive semi-definite.

2. The Fourier transform of $f$ has constant modulus (i.e. $|\hat{f}(x)|=$ const) if and only if $f$ is orthogonal to its translations:

$$
\langle f, \hat{x}(q) f\rangle=\sum_{x} \bar{f}(x) f(x-q)=0,
$$

for all non-zero $q \in \mathbb{Z}_{d}$.

Proof. The matrix $A$ is circulant. It is well-known that circulant matrices are normal (hence diagonalizable) with eigenvalues given by the Fourier transform of the first row (up to a positive normalization constant). The first claim is now immediate.

By the same argument, $A$ is proportional to a unitary matrix if and only if $|\hat{f}(q)|$ is constant. But a matrix is unitary if and only if its rows form an ortho-normal set of vectors.

The next three lemmas harvest some consequences of Bochner's Theorem to gain information on the pointwise modulus $|\psi(q)|$ of the vector.

Lemma 4. (Modulus Inequality) Let $\psi$ be a state vector with positive Wigner function.

It holds that

$$
|\psi(q)|^{2} \geq|\psi(q-x)||\psi(q+x)|
$$

for all $q, x \in \mathbb{Z}_{d}$.
Proof. Fix a $q \in \mathbb{Z}_{d}$. As $W_{\psi}$ is non-negative, so is the Fourier transform of $K_{\psi}(q, x)$ with respect to $x$. Bochner's Theorem implies that $A^{x} y=K(x-y, q)$ is positive semi-definite $(p s d)$ which in turn implies that all principal sub-matrices are psd. In particular the determinant of the $2 \times 2$ principal sub-matrix

$$
\begin{aligned}
& \left(\begin{array}{cc}
K_{\psi}(q, 0) & K_{\psi}(q, 2 x) \\
K_{\psi}(q,-2 x) & K_{\psi}(q, 0)
\end{array}\right) \\
= & \left(\begin{array}{cc}
|\psi(q)|^{2} & \psi(q+x) \bar{\psi}(q-x) \\
\bar{\psi}(q+x) \psi(q-x) & |\psi(q)|^{2}
\end{array}\right)
\end{aligned}
$$

must be non-negative. But this means

$$
|\psi(q)|^{4}-|\bar{\psi}(q+x) \psi(q-x)|^{2} \geq 0,
$$

which proves the theorem.

We will call the set of points where a state-vector is non-zero its support.

Lemma 5. (Support Lemma) Let $\psi$ be a state vector with positive Wigner function.

If $\psi$ is supported on two points, then it has maximal support.

Proof. Denote by $S=\operatorname{supp} \psi$ the support of $\psi$. $S$ has the property to contain the midpoint of any two of its elements. Indeed, if $a, b \in S$, then setting $q=2^{-1}(a+b)$ and $x=2^{-1}(a-b)$ in the Modulus Inequality shows that

$$
\left|\psi\left(2^{-1}(a+b)\right)\right| \geq|\psi(a)||\psi(b)|>0,
$$

hence $2^{-1}(a+b) \in S$.

Assume there exist two points $a, b \in S$. Requiring $a=0$ is no loss of generality, for else we substitute $\psi$ by $\psi^{\prime}=w(0,-a) \psi$. By Eq. (2), $\psi^{\prime}$ has positive Wigner function if and only if $\psi$ has.

We claim that

$$
2^{-l} \beta b \in S
$$

for all $l$ and $\beta \leq 2^{l}$. The proof is by induction on $l$. Suppose Eq. (3) holds for some $l$. If $\beta \leq 2^{l+1}$ is even, then $2^{-l-1} \beta b=2^{-l}(\beta / 2) b \in S$. Else,

$$
2^{-l-1} \beta b=2^{-1}\left(2^{-l} \frac{\beta-1}{2} b+2^{-l} \frac{\beta+1}{2} b\right) \in S,
$$

which proves the claim.

Now, by Fermat's Little Theorem $2^{d-1}=1 \bmod d$ and hence, setting $l=d-1$ in Eq. (3), we conclude that $\beta b \in S$ for all $\beta \leq d-1$. But every point in $\mathbb{Z}_{d}$ is of that form.

Lemma 6. (Constant Modulus) Let $\psi$ be a state vector with positive Wigner function and maximal support.

Then $|\psi(q)|=$ const. 
Proof. Pick two points $x, q \in \mathbb{Z}_{d}$ and suppose $|\psi(q)|>$ $|\psi(x)|$.

Letting $z=x-q$, the assumption reads $|\psi(q)|>\mid \psi(q+$ $z) \mid$. Lemma 4 centered at $q+z$ gives

$$
\begin{aligned}
|\psi(q+z)|^{2} & \geq|\psi(q)||\psi(q+2 z)| \\
& >|\psi(q+z)||\psi(q+2 z)|,
\end{aligned}
$$

therefore $|\psi(q+z)|>|\psi(q+2 z)|$. By inducting on this scheme, we arrive at

$$
|\psi(q)|>|\psi(q+z)|>|\psi(q+2 z)|>\cdots
$$

and hence $|\psi(q)|>|\psi(q+d z)|=|\psi(q)|$, which is a contradiction.

Thus $|\psi(q)| \leq|\psi(x)|$. Swapping the roles of $x$ and $q$ proves that equality must hold.

Theorem 7. (Main Theorem - Special Case) Let $d$ be prime and $\psi \in L^{2}\left(\mathbb{Z}_{d}\right)$ be a state vector with positive Wigner function. Then $\psi$ is a stabilizer state.

Proof. By the Support Lemma, $\psi$ is either a position eigenstate or else it has maximal support. In the former case, $\psi$ is manifestly a stabilizer state, so we need only treat the latter. Let $U$ be a Clifford operation. Since $U$ preserves positivity, the Support Lemma applies to $U \psi$. Suppose $U$ is such that $\operatorname{supp} U \psi$ contains just a single point. Then $U \psi$ belongs to the computational basis and hence, by definition, $\psi$ is a stabilizer state.

Therefore, we are left to treat those state vectors whose image under any Clifford operation has maximal support. The proof is concluded by showing that such states do not exist.

For assume there is such a vector $\psi$. As $\psi$ has pointwise constant modulus, so does $K_{\psi}$. Employing Theorem 3. we find that, for every fixed $q_{0}, W\left(p, q_{0}\right)$ is orthogonal to its own translations. But since $W$ is non-negative, it follows that $W\left(p, q_{0}\right)$ can be non-zero on at most one point. A Wigner function that is concentrated at a single point can not represent a physical state [23]. There must hence exist at least two points $a, b$ in the support of $W$ (note that we are now considering the support of Wigner functions and no longer the support of state vectors). Making once more use of the fact that translations are implemented by Clifford operations, assume $a=0$. There exists a unit-determinant matrix $S$ that sends $b$ to a vector of the form $S b=\left(0, q_{0}\right)^{T}$. But then there are two points in the support of $W_{\mu(S) \psi}\left(p, q_{0}\right)$, contradicting our earlier derivation.

\section{SUMMARY}

We have proved a 'classicality result' for discrete Wigner functions: those state vectors which give rise to a classical probability distribution in phase space belong to the set of stabilizer states. These, in turn, allow for an efficient classical description. Comparing the proof of the special case treated here to the involved argument employed in Ref. [5], it becomes apparent how much the geometrical properties of integer residues modulo prime numbers simplify the structure.

\section{ACKNOWLEDGMENTS}

The author is grateful for support and advice provided by Jens Eisert during all stages of this project. Comments by and discussions with K. Audenaert S. Chaturvedi, H. Kampermann, M. Kleinmann, A. Klimov, M. Ruzzi, and C.K. Zachos are kindly acknowledged.

This work has benefited from funding provided by the European Research Councils (EURYI grant of J. Eisert), the European Commission (Integrated Project QAP), the EPSRC (Interdisciplinary Research Collaboration IRCQIP), and the DFG.
[1] R.L. Hudson, Rep. Math. Phys. 6, 249 (1974).

[2] F. Soto and P. Claverie, J. Math. Phys 24, 97 (1983).

[3] E.F. Galvao, Phys. Rev. A 71, 042302 (2005); C. Cormick, E.F. Galvao, D. Gottesman, J. Pablo Paz, and A.O. Pittenger, Phys. Rev. A 73012301 (2006).

[4] W.K. Wootters, Ann. Phys. NY 176, 1 (1987).

[5] D. Gross, J. Math. Phys. 47, 122107 (2006).

[6] A. Vourdas, Rep. Prog. Phys. 67, 267 (2004).

[7] C. Miquel, J.P. Paz, and M. Saraceno, Phys. Rev. A 65, 062309 (2002).

[8] C.A. Munoz Villegas, A. Chavez Chavez, S. Chumakov, Yu. Fofanov, A.B. Klimov, quant-ph/0307051.

[9] A.B. Klimov, C Muñoz, J. Opt. B, 7, S588 (2005).

[10] M. Ruzzi, D. Galetti, M.A.. Machiolli, J. Phys. A, 38, 6239 (2005).

[11] S. Chaturvedi E. Ercolessi, G. Marmo, G. Morandi, N.
Mukunda, R. Simon, J. Phys. (Pramana), 65, 981 (2006).

[12] D. Gross. Diploma Thesis. University of Potsdam (2005). Available online at http://gross.qipc.org.

[13] K.S. Gibbons, M.J. Hoffman, and W.K. Wootters, Phys. Rev. A 70, 062101 (2004).

[14] U. Leonhardt, Phys. Rev. A 53, 2998 (1996).

[15] M.A. Nielsen, I.L. Chuang, Quantum computation and quantum information. (Cambridge Univ. Press, Cambridge, 2000).

[16] F.J. Narchowich, J. Math. Phys. 29, 2036 (1988).

[17] T. Bröcker and R.F. Werner, J. Math. Phys. 36, 62 (1995).

[18] A. Weil, Acta Mathematica 111, 143 (1964).

[19] D. Gottesman, quant-ph/9807006

[20] Note that the boundedness of $\psi \in L^{2}\left(\mathbb{R}^{n}\right)$ implies that $\theta$ has positive semi-definite imaginary part. 
[21] There is a confusing similarity of names: the Weil representation (after André Weil) acts on the Weyl operators (after Hermann Weyl).

[22] Note that the "Clifford group" which appears in the context of quantum information theory [19] has no connection to the group by the same name used e.g. in the representation theory of $S O(n)$.

[23] Such a Wigner function corresponds to a Hermitian operator with both positive and negative eigenvalues (see Ref. [5]). One can think of this fact as an incarnation of the uncertainty principle. 